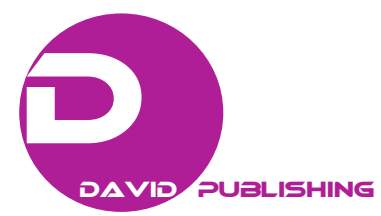

\title{
Modeling of HIV Transmission in Nasarawa State, Nigeria: An Analysis of Distribution of New Infections
}

\author{
Ishaku Ara Bako ${ }^{1}$, Abdulsamad Salihu ${ }^{2}$, Ifeanyi Okekearu ${ }^{2}$ and Jennifer Anyanti ${ }^{2}$ \\ 1. Department of Epidemiology and Community Health, College of Health Sciences, Benue State University, Makurdi, Benue State, \\ Nigeria \\ 2. Society for Family Health (SFH), Abuja, FCT 970101, Nigeria
}

\begin{abstract}
The need to understand local HIV epidemics and linking the prevention and other interventions to evidences become very important for the success of HIV response. The objective of the study was to estimate the distribution of new HIV infections among adult population (15-49 yrs) and to identify the groups at highest risk of HIV infection in Nasarawa State Nigeria to inform HIV prevention programme planning and Implementation. The study was based on the Modes of HIV Transmission (MOT) incidence model recommended by the Joint United Nation's Programme on AIDS (UNAIDS). Persons aged 15-49 years were divided into independent groups based on their risky behaviours. Demographic, epidemiological and behavioral data were obtained for each risk group from available survey reports/documents and inputed into the UNAID's MOT Model spreadsheet.The model estimated that more than 45\% of new infections would occur amongst persons who reported "low risk" sex. The Injecting Drug Users, Female Sex Workers (FSW) and Men having Sex with Men and their partners were estimated to contribute $20.7 \%$. Persons reporting low risk sex practices, a sub-population that includes cohabiting or married sexual partners need to be targeted with appropriate HIV prevention interventions such as HIV Counselling and Testing, condom promotion, Interpersonal communications and other partner reduction strategies.
\end{abstract}

Key words: Risk group, incidence, modes of transmission.

\section{Introduction}

The HIV/AIDS epidemic is now in its 3rd decade with an estimated 34 million people living with HIV worldwide at the end of 2011. Sixty percent of all people living with HIV reside in sub-Saharan Africa. Nigeria has the second largest population of persons living with HIV in the World with an estimated 3.1 million living with the disease [1]. Nasarawa state is one of the states with high HIV/AIDS prevalence in Nigeria. Estimates from Nigeria's antenatal clinic HIV sentinel survey shows the state has HIV prevalence of $7.5 \%$ compared to the national average of $4.1 \%$ [2]. One of the factors fuelling the epidemic in Nasarawa state its proximity to Abuja, Nigeria's federal capital. This results to the influx of migrant populations into the state.

Corresponding author: Ishaku Ara Bako (MBBS, FMCPH), senior lecturer, research fields: epidemiology, medical statistics, health services management.
HIV prevention interventions need to be targeted at populations that are experiencing the highest burden of new (incident) infections. The Joint United Nation's Programme on AIDS' (UNAIDS) Modes of Transmission (MOT) model uses national prevalence and behavioural data to model the distribution of incidence in key risk populations. It is an important tool in supporting country and sub-national teams to understand their epidemics to enable them make better decisions on prioritization and definition of goals and targets for effective scale up to Universal Access [3]. The UNAIDS in collaboration with the World Bank in 2007 assisted some countries in East and Southern Africa to successfully apply the Incidence by Modes of Transmission Model [4-6]. This was followed by analysis in West African countries including Nigeria in 2008 [7-10]. Reports have indicated that substantial heterogeneity exists within a country necessitating that local data on HIV epidemic be used to inform local 
HIV interventions [11]. However, there is lack of information on HIV incidence to inform HIV and AIDS prevention interventions [12-14].

The Modes of HIV analysis in Nasarawa State was an attempt to apply the UNAIDS' model to define the pattern of new HIV infection at a sub national level. It was aimed at understanding the local epidemic in Nasarawa State using available HIV prevalence, demographic and behavioural data and thus to improve the scope, relevance and comprehensiveness of the state HIV prevention efforts. Specifically, the Modes of Transmission review was to identify the distribution of the most recent infections and the populations at greatest risk for infection through incidence modelling and to make recommendations for prevention, policy and programmatic actions to ensure a stronger and more effective state level prevention strategy.

\section{Materials and Methods}

The project was conducted by a state team, supported by the Department for International Development-funded Enhancing Nigeria's Response to HIV and AAIDS Programme (DFID/ENR) programme with guidance from an MOT modelling consultant.

\subsection{Modes of Transmission Model}

The purpose of the model was to calculate the expected short-term (one year) incidence of HIV infections among the adult population by mode of transmission. The calculation was based on the current prevalence of HIV infection, the number of individuals in particular risk groups, and the risk of exposure to infection within each group. The model utilized a spreadsheet based on the UNAIDS Reference Group on Estimates, Modelling and Projections [15].

Key documents that provided inputs for the model include the following: 2005 and 2008 Antenatal Care Serological Surveillance Surveys, the Nigerian Demography and Health Survey (NDHS) 2008, the Nigerian HIV/AIDS and Reproductive Health Survey
(NARHS) 2003 and 2005, the National Behavioural Surveillance Survey (BSS) 2005, the country's first Integrated Bio-Behavioural Surveillance Survey (IBBSS) 2007 and the country's first population based HIV sero-prevalence surveillance survey (NARHS plus 2007). Where Nasarawa State representative data did not exist, North Central geopolitical zonal values were used or results of surveys that included proximal states. Where no zonal or proximal data were not available, reliable in-country research findings were used.

These values were then shared with stakeholders within the State through a stakeholders' workshop and externally through correspondence for input and consensus. These inputs were used to finalise the model.

\subsection{Inputs}

2.2.1 Percentage of Population with Risk Behaviour

The adult (15-49 years) population in Nasarawa was divided into groups based on their highest risk factor from the main transmission modes in the country: sexual transmission (heterosexual and homosexual) and sharing needles during intravenous drug use.

Initially the general adult (15-49 years) population, disaggregated by sex, was differentiated by their sexual activity over the last 12 months. Based on data from the NDHS 2008, and NARHS plus 2007, they were divided into those that did not have sex in the last 12 months; those that had sex but with only marital partner and those that had non-marital sex. For this assessment, the definition of commercial sex was agreed to be the "exchange of gifts or money for sex".

The percentage of men who have sex with men (MSM) was determined from the high-risk group surveys (IBBSS 2007) conducted in the country. The fraction of persons that reported injecting drug use was determined from the general population behavioural survey (NARHS 2003).

2.2.2 Sexual Partners of Persons within High Risk Groups 
The MOT model aims to show the effect of risk not only from the perspective of the person who has sex or other risk behaviours associated with HIV infection, but also the effect of their behaviours on their sexual partners.

(1) Sexual partners of male injecting drug users: To estimate the percentage of females who were stable partners of IDUs, the percentage of IDUs who reported living with their sexual partners, independently of whether they were married or not, was multiplied by the percentage of the male population who were IDUs: this was equal to $0.25 * 0.4=0.10 \%$. This information was taken from the IBBSS 2007.

(2) Sexual partners of female injecting drug users: Unfortunately, the IBBSS did not provide reliable information on female IDUs' sexual characteristics. We were more interested in regular sexual partners of female IDUs. We made an approximation, that $70.6 \%$ of female partners of IDU were either married or living with their partners. This was because in the NHDS 2008 , this percentage of women was reported to be in marital or cohabiting union.

(3) Sexual partners of MSM: This was obtained from percentage of MSM were married or living with partners.

(4) Sexual partners of clients of Female sex workers: Partners of clients of sex workers were estimated from the percentage of clients of sex worker who were married according to the NDHS. These partners of FSW clients were hitherto assumed to have been low risk.

(5) Sexual partners of men and women engaging casual heterosexual sex: To estimate the number of persons who were engaging in casual sex we assumed the default values in the model i.e. that $80 \%$ of persons engaging in casual sex had a regular partner.

\subsection{HIV Prevalence}

Prevalence amongst the general population was based on the 2007 NARHS plus population based sero-prevalence survey conducted in the country.
Nasarawa state had a prevalence of $6.8 \%$. The prevalence amongst the high-risk groups was based on the 2007 Integrated Behavioural and Biological survey conducted amongst High Risk groups in Nigeria. The prevalence amongst partners of high-risk groups (partners of FSWs) was estimated by studying the relationship between sexual behaviour and HIV prevalence in West African countries and limited research in Nigeria and deducing the possible prevalence amongst these groups based on the prevalence amongst the general population and the high risk groups.

\subsection{STI Prevalence}

Sexually Transmitted Infections (STIs) are known to affect the rate of transmission of HIV during sex. The prevalence of STI in the population and its distribution amongst the various groups will affect the rate of HIV transmission through sex. The prevalence of infection was estimated by the percentage of the various groups that reported having unusual genital discharge or a genital ulcer in the last 12 months. The percentage for the general population was obtained from the 2008 NDHS while the percentages for the high-risk groups were obtained from the IBBSS 2007.

\subsection{Number of Sexual Partners and Acts per Partner per Year}

An assumption was made that the average sexually active person had about 100 acts of sexual intercourse a year as in the latest NDHS surveys where this question was asked the reported number varied between 50 and 100 sex acts per year.

According to the IBBSS 2007, brothel based FSW and non-brothel based FSW had on average 34 and 25 clients per week respectively. As the sample, sizes of these two groups were quite close it was assumed that each represented half of the FSW population and it was estimated that FSW had an average of 2 clients per day for 20 days. Assuming, FSW take 12 weeks off due to menstrual periods. Their total number of clients per 
year was: 600 .

The number of sex partners per year was calculated by determining the average number of partners that people in each group had based on their responses to questions in the various surveys conducted in the country. People reporting no sexual activity in the last 12 months were presumed to have no sexual partner; those reporting only marital sex were presumed to have only one sexual partner and those stating that they had had non-marital sex were assumed to have more than one.

\subsection{Percentage of Acts Protected (\%)}

These are the number of sex acts in which the persons took precaution against HIV infection by using a condom; this is approximated by determining the percentage of last acts of sex in which a condom was used. These were obtained from the IBBSS and the NDHS 2003 \& NARHS 2005. Condom use among persons in the low risk group was presumed to be the same for the state as the current use of condoms reported in the NARHS+ 2007 for North Central zone.

\section{Results}

Table 1 Incidence of HIV infections in one year among adults in Nasarawa Nigeria, 2010.

\begin{tabular}{lllll}
\hline Adult risk category & Total number with risk behaviour & Incidence & \% of incidence & Incidence per 100,000 \\
\hline Injecting Drug Use (IDU) & 1,303 & 201 & 6.04 & 15,461 \\
$\quad$ Partners IDU & 600 & 6 & 0.17 & 956 \\
Sex workers & 4,344 & 61 & 1.82 & 1,394 \\
$\quad$ Clients & 16,508 & 164 & 4.92 & 993 \\
$\quad$ Partners of Clients & 5,613 & 42 & 1.26 & 750 \\
MSM & 3,041 & 205 & 6.13 & 6,728 \\
$\quad$ Female partners of MSM & 1,186 & 13 & 0.39 & 1,107 \\
Casual heterosexual sex & 152,919 & 328 & 9.83 & 214 \\
$\quad$ Partners CHS & 122,335 & 727 & 21.79 & 594 \\
Low-risk heterosexual & 318,598 & 1,558 & 46.71 & 489 \\
No risk & 242,412 & 0 & 0.00 & 0 \\
Medical injections & 868,860 & 11 & 0.32 & 1 \\
Blood transfusions & 8,689 & 20 & 0.61 & 234 \\
Estimated total adult population & 868,860 & 3,335 & 100.00 & 384 \\
\hline
\end{tabular}

The model estimated that 3,335 new infections would occur in the following one year amongst the 15-49 years adult population (Table 1).

The exposure groups with the highest incidence risk were persons who reported low risk sex in the previous year. They were estimated to account for about $47 \%$ of all new infections. About 23\% of infections would occur amongst people who are sexual partners of high-risk groups (female sex partners of MSM; partners of IDUs, partners of clients of female sex workers and partners of persons who have casual high-risk sex (Table 1, Fig. 1).

Directly, IDU, FSW and MSM with their partners, contribute as much as $21 \%$ of new infections. New infections arising from commercial (transactional) sex accounted for $8 \%$ of new cases, of which of clients dominated with $4.9 \%$ of all new cases.

Persons who had casual sex and their partners accounted for about $32 \% \quad(9.8 \%$ and $21.8 \%$ respectively) of new infections. Medical injections and blood transfusion together accounted $0.9 \%(0.32 \%$ and $0.61 \%$ respectively) (Fig. 1). 


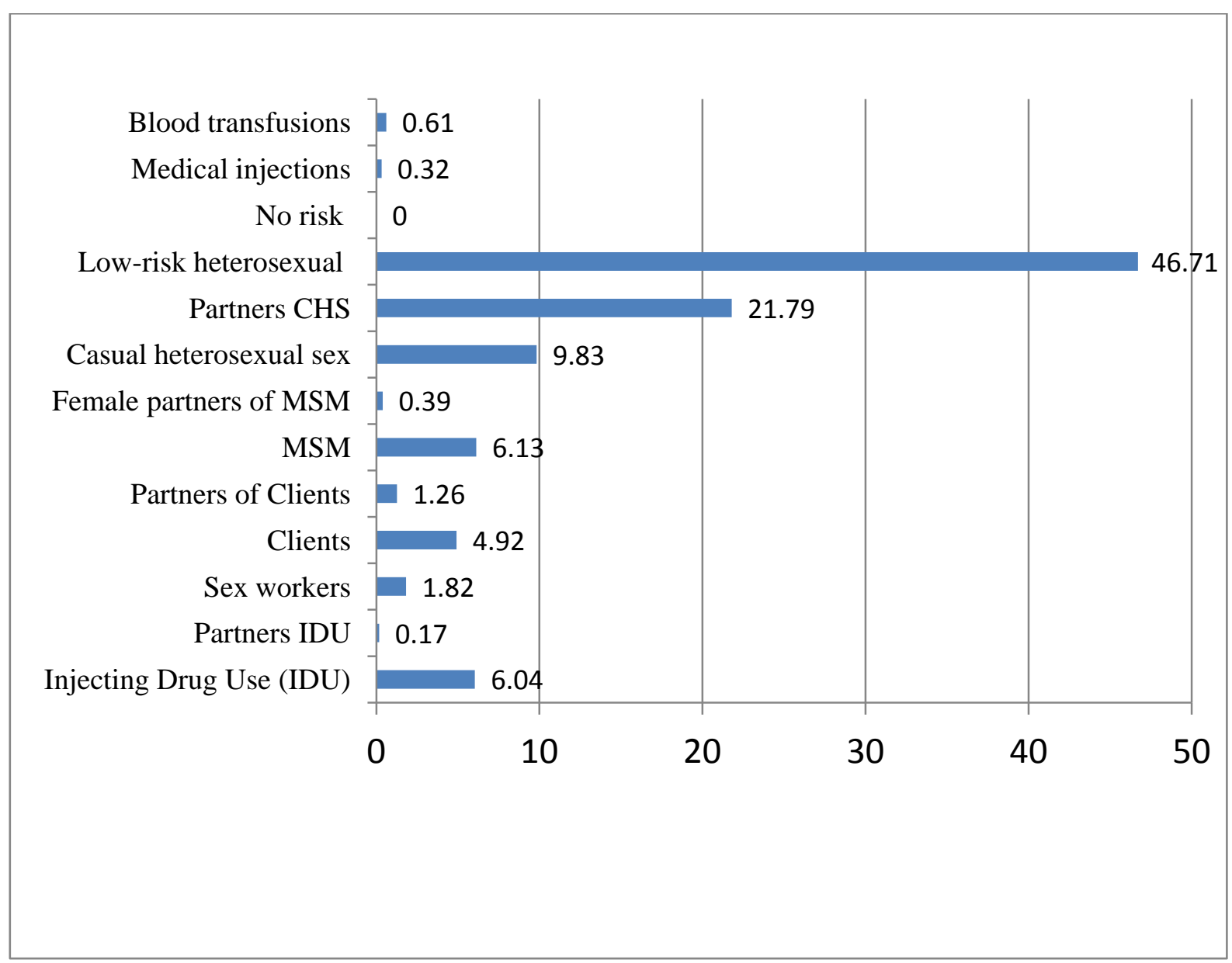

Fig. 1 Estimated Proportion of New HIV Infection among adult risk groups population in Nasarawa State Nigeria, 2010.

\section{Discussion}

More than $45 \%$ of the infections occur amongst persons practicing "low risk" sex. This finding is higher than what was found from other countries and National estimates from Nigeria. In the West African sub region, about $30 \%$ of all new infections occur in people who have low risk behaviour. It was estimated to be $36 \%$ for Nigeria, 28\% (Benin), 28\% (Ghana), and $24 \%$ in Côte d'Ivoire [15], 44.1\% (Kenya) [16], 56\% (Iran) [17]. This could be accounted for by among other reasons low HCT rate; only $36.1 \%$ of men and $34.1 \%$ of women in the state ever tested for HIV [18]. HIV infection acquired because of previous or present high-risk behaviours or relationships by one of the sex partners is easily transmitted to unsuspecting partners. The presumed perceived low risk of infection amongst persons who themselves are keeping to one partner faithfully, needs to be addressed. The notion that one is safe because he or she is doing the right thing needs to be revised to educate people that they are not safe until their partners are also faithful. There is a need to ensure that people are aware of the HIV status and the sexual practices of their partners. Any form of doubt needs to be addressed through the use of condoms during sex.

About 24\% of new HIV infections were estimated to occur amongst people who are sexual partners of high-risk groups (female sex partners of MSM; partners of IDUs, partners of clients of female sex workers and partners of persons who have casual high-risk sex). This finding did not agree with a previous study which showed that in North Africa and Middle East, Sex workers and IDUs contribute the highest proportion of new HIV infection [19]. In Kenya, 
sex workers and their clients, IDUs, MSM and prison population contribute $33 \%$ of new infections [20]. Partners of CHS, IDUs and of FSWs' clients are people who ordinarily should have been classified as low risk but are now at a higher risk of acquiring HIV infection because of their relationships with people known to practice high risk sex and therefore not very visible as high-risk groups.

In spite of the fact that the majority of the infections were due to the HIV transmission amongst the general population, the high-risk groups still contribute a significant portion of the new HIV infections. Directly, IDU, FSW and MSM with their partners, contribute as much as $21 \%$ of new infections. This is quite significant because these groups constitute only about $3 \%$ of the adult population.

The modeling exercise in Nasarawa State has brought out a number of limitations in the application of the UNAIDS Model particularly at a sub-national level. The Model does not take into account the heterogeneity in risk behaviours within each risk group as individuals were placed in groups considered to be the highest risk. However, effect of multiple exposures in same individuals on HIV transmission probability may be higher than effect of the highest risk alone. In addition, there was limited state specific data at the time of the modeling necessitating the use data from contiguous states or zonal averages which may not be exactly true for the state. As new data become available and because the pattern of HIV transmission changes over time [14], it is imperative that MoT analysis is repeated on a regular basis. Finally, the analysis used findings from surveys which relied on self reported behaviours with possibilities of bias. However, the exercise provided an opportunity to build the capacity of stakeholders from the state on MOT analysis and as more data become available, subsequent exercise would be conducted with minimal support from outside the state. The findings from this study could also be used as an advocacy tool to government and donors to solicit for appropriate funding for the state HIV prevention interventions.

HIV Counselling and Testing (HCT) needs to be scaled up rapidly to the general population in the state. Efforts must be made to get couples to undergo couple HCT. Condom use should be socially marketed to be the norm in any sexual relationship especially where the partners don't know their HIV status. There is need to overcome all barriers to condom usage including socio-cultural factors and poor availability. Opportunities for mass campaign and social movement should be explored with religious and community leaders to discourage multiple partnering as a threat to individual and public health. Community based HIV prevention interventions such as Interpersonal Communications (IPC), Society Tackling AIDS through Rights (STAR), Peer Education Plus (PEP) model, Priority for Local AIDS Control Efforts (PLACE) and Voice For Humanity (VFH) which has already been piloted in the state should be scaled up rapidly to increase HIV knowledge and effect appropriate behavioural change among the general population. Budgeting for HIV Prevention should take distribution of new infections into consideration and based on the relative magnitude of the mode of transmission. There is need to carry out state specific surveys to estimate the size and characteristics of injecting drug users, men having sex with men and their partners as well as clients of sex workers to use for future MOT analysis. National HIV related biological and behaviours surveys should be disaggregated by state to enable findings to be more useful at the state level.

\section{References}

[1] UNAIDS. 2012. World AIDS Day Report. Joint United Nations Programme on HIV/AIDS (UNAIDS).

[2] Federal Ministry of Health, Department of Public Health, National AIDS/STI Control Programme. 2010. Technical report 2010: National HIV/Syphilis sero-prevalence sentinel survey among pregnant women attending antenatal clinics in Nigeria. Abuja.

[3] UNAIDS. 2007. Modelling the Expected Short term Distribution of Incidence of HIV Infections by Exposure 
Group, Manual. Joint United Nations Programme on HIV/AIDS (UNAIDS).

[4] Gelmon, L., Kenya, P., Oguya, F., Cheluget, B., and Haile, G. 2009. Kenya: HIV Prevention Response and Modes of Transmission Analysis Report. Kenya National AIDS Control Council, UNAIDS, World Bank Global HIV/AIDS Program/Global AIDS M\&E Team.

[5] Khobotlo, M., Teshelo, R., Nkonyana, J., Ramoseme, M., Khobotle, M., Chitoshia, A., Hildebrand, M., and Fraser, N. 2009. Lesotho: Analysis of Prevention Response and Modes of Transmission Study, Kingdom of Lesotho, UNAIDS, World Bank Global HIV/AIDS Program Report.

[6] Wabwire-Mangen, F., Odiit, M., Kirungi, W., Kawaeesa Kisitu, D., and Okara Wanyama, J. 2009. Uganda: HIV Modes of Transmission and Prevention Response Analysis Report, Uganda AIDS Commission, UNAIDS.

[7] UNAIDS. 2010. New HIV Infections by Mode of Transmission in West Africa: A MultiCountry Analysis. UNAIDS

[8] Shubber, Z., Mishra, S., Vesga, J. F., and Boily, M. C. 2014. "The HIV Modes of Transmission Model: A Systematic Review of Its Findings and Adherence to Guidelines." J. Int. AIDS Soc. 17 (1): 18928.

[9] National AIDS Control Agency (NACA)/UNAIDS. 2010. Modes of HIV Transmission in Nigeria, An Analysis of the Distribution of New HIV infections in Nigeria and Recommendations for Prevention.

[10] Bosu, W. K., Yeboah, K., Gurumurthy, R., and Atuahene, K. 2009. "Modes of Transmission in West Africa: Analysis of the Distribution of New HIV Infections in Ghana and Recommendations for Prevention Report." Ghana AIDS Commission, UNAIDS.

[11] Case, K. K, Ghys, P. D., Gouws, E., Eaton, J. W., Borquez, A., Stover, J., Cuchi, P., Abu-Raddad, L. J., Garnett, G. P., and Hallett, T. B. 2004. "Understanding the Modes of Transmission Model of New HIV Infection and Its Use in Prevention Planning." Sex Transm Infect. 80 (Suppl 1): i19-24.

[12] FMOH Nigeria. 2006. Technical report 2005: National HIV/Syphilis sero-prevalence sentinel survey among pregnant women attending antenatal clinics in Nigeria. Federal Ministry of Health, Department of Public Health, National AIDS/STI Control Programme. Abuja.

[13] FMOH Nigeria. 2008. Technical report 2008: National HIV/Syphilis sero-prevalence sentinel survey among pregnant women attending antenatal clinics in Nigeria. Federal Ministry of Health, Department of Public Health, National AIDS/STI Control Programme. Abuja.

[14] Pisani, E., Garnett, G. P., Grassly, N. C., Brown, T., Stover, J., Hankins, C., Walker, N., and Ghys, P. D. 2003. "Back to Basics in HIV Prevention: Focus on Exposure." BMJ 326 (7403): 1384-7.

[15] Joint United Nations Programme on HIVIAIDS (UNAIDS). 2009. New HIV Infections by mode of transmission in West Africa: A Multi-Country Analysis: 2006 Report.

[16] Kenya National AIDS Control Council. 2009. Kenya HIV Prevention and Mode of HIV Transmission Analysis, Final Report.

[17] Nasirian, M., Doroudi, F., Gouya, M. M., Sedaghat, A., and Haghdoost, A. A. 2012. "Modeling of Human Immunodeficiency Virus Modes of Transmission in Iran." J. Res. Health Sci. 12: 81-7.

[18] DFID/ENR. 2011. Nasarawa State HIV and AIDS Sexual and Reproductive Health Knowledge, Attitude and Behavioural Survey. Abuja, Nigeria.

[19] Gous, E., and Cuchi, P. 2012. "Focusing on the HIV Response through Estimating the Major Modes of HIV Transmission: A Multi Country Analysis." Sex Transm. Inf. 88 (Suppl 2): i76-i85.

[20] Gouws, E., White, P. J., Stover, J., and Brown, T. 2006. "Short Term Estimates of Adult HIV Incidence by Mode of Transmission: Kenya and Thailand as Examples." Sex Transm. Inf. 82 (Suppl 3): iii51-5. 